\title{
Coronavirus and Cardiovascular System
}

\author{
Sandeep Parekh ${ }^{1} \quad$ Kewal Krishan Talwar ${ }^{1}$ \\ ${ }^{1}$ Department of Cardiology, Max Super Specialty Hospital Mohali, \\ Mohali, Punjab, India
}

\begin{abstract}
Address for correspondence Kewal Krishan Talwar, MD, DM, DSc, C-625, Second Floor, New Friends Colony, New Delhi 110025, India (e-mail: kktalwar@hotmail.com).
\end{abstract}

Ann Natl Acad Med Sci (India):2020;2:99-101

\begin{abstract}
With the pandemic of COVID-19 infection spreading throughout the world leading to unprecedented serious burden on healthcare, our understanding of the virus continues to grow on a daily basis with the whole world united to find a vaccine/cure to combat the disease. Clinical experience with growing number of cases has provided us with insight into the pathophysiology of the disease and the various systems of the body it affects. The growing experience shows that cardiovascular complications occur in significant number of cases and contribute to increased mortality. The complications and mortality is also higher in those with pre-existing cardiovascular disease. The pathogenesis of increased cardiac complications is possibly related to the action of virus surface spike proteins through ACE2 receptors expressed in the myocardium.

Keywords

- coronavirus

- COVID-19

- cardiovascular disease Although various drugs/therapies are being tried currently, no effective therapy is still available. It is emphasized that those with existing cardiac disease should optimally take their medicines, and religiously follow preventive measures like social distancing, self-isolation and hand hygiene.
\end{abstract}

\section{Epidemiology of Cases}

On December 31, 2019, 27 cases of pneumonia of unknown cause first surfaced in Wuhan, Hubei province, which were reported to the World Health Organization (WHO) country office in China. Investigators in Wuhan published their initial experience with this infection in the recent March edition of Journal of the American Medical Association (JAMA). ${ }^{1}$ After identification and isolation of the pathogen, this virus was originally called 2019 novel coronavirus (2019-nCoV) and officially renamed severe acute respiratory syndrome CoronaVirus 2 (SARS-CoV-2) by the WHO. ${ }^{2}$ The virus has since then spread throughout the world with the first case in India reported on January 30, 2020 in Thrissur, Kerala. Taking into cognizance the alarming rate of spread and severity of the disease, WHO characterized COVID-19 as a pandemic on March 11, 2020. To date, nearly 5 million people have been reported to be infected worldwide, with over 300000 deaths, and the numbers are rapidly rising every day.

Although the main symptoms of COVID-19 appear to be respiratory, some infected patients suffer, in addition, from severe cardiovascular damage. This is especially true for patients with underlying cardiovascular diseases who are more prone to infection and might have an increased risk of death. ${ }^{3}$

\section{Pathophysiology of COVID-19 Infection}

Angiotensin-converting enzyme 2 (ACE2) is a membrane-bound aminopeptidase which is highly expressed in the heart and lung. Infection is triggered by the binding of the spike protein of the virus to ACE2 and consequent invasion of lung (alveolar epithelial cells) and heart, resulting in respiratory and cardiac complications. ACE2 is a homolog of ACE and converts angiotensin II to angiotensin 1-7. It thus protects the heart/lungs from the harmful effect of angiotensin II which is mediated via systemic vasoconstriction. The exact mechanism of cardiac involvement is speculated to be either direct myocardial damage or via an imbalance between subtypes of Thelper cells, leading to a cytokine storm and shocklike state.

Cardiovascular complications occur in a significant proportion of patients and are an important independent risk factor for mortality. It has been observed that there is a vulnerable population prone to develop serious complications, 
which includes patients with comorbid conditions, namely, advanced age, diabetes mellitus, hypertension, coronary artery disease, heart failure, chronic lung disease, and patients with cancer or with immune compromised status. ${ }^{4}$

\section{Cardiovascular Manifestations}

\section{Myocarditis Related Myocardial Injury}

The severity of myocardial involvement after COVID-19 infection varies, with patients presenting with chest pain, arrhythmias, dyspnea or acute left ventricular (LV) failure. ${ }^{1,3}$ The electrocardiogram (ECG) abnormalities include nonspecific ST-T abnormalities, T wave inversions, PR segment and ST segment depression, and elevations mimicking acute coronary syndrome (ACS) in some cases. Differentiating ACS from COVID-19 related myocarditis by ECG and clinical presentation may be challenging. Echocardiography may be helpful in this situation, with regional wall motion abnormality demonstrated in ACS, and either no wall motion abnormality or global hypokinesia seen in COVID-19 related myocarditis.

Acute myocardial injury associated with COVID-19 mainly manifests as an increase in high-sensitivity cardiac troponin I (hs-cTn I), requiring admission to the intensive care unit (ICU). Patients with severe symptoms often have complications involving acute myocardial injury. ${ }^{1}$ ECG, echocardiography abnormalities and troponin elevation correlate with severity of the infection and denote worse outcomes in patients with severe infection. ${ }^{5,6}$ There is usually raised CRP, CD4, CD8 and interleukin 6 (IL-6), pointing to an inflammatory response. This inflammatory response may result in myocarditis. Hu et al reported a patient with fulminant myocarditis, following SARS-CoV-2 infection. The patient showed remarkable improvement in LV function with methylprednisolone and immunoglobulins in addition to other symptomatic therapy. ${ }^{7}$

\section{Acute Coronary Syndrome}

Some patients with SARS-Cov-2 present with ACS and ST-elevation myocardial infarction (STEMI) or non-STEMI picture.

Treatment of STEMI in COVID-19 patients is controversial in nature. In patients presenting with "low-risk STEMI," defined as inferior STEMI without right ventricular involvement or lateral wall MI and also without hemodynamic compromise, thrombolytic therapy has been suggested to be the preferred option by American College of Cardiology (ACC) when compared with percutaneous coronary intervention (PCI) to avoid exposure to staff. If PCI is pursued, appropriate personal protective equipment (PPE) for staff is mandatory, with full decontamination of the catheterization laboratory after completion of the procedure. ${ }^{8}$

In a recent study published in Circulation, Stefanini et $\mathrm{al}^{9}$ reported a review of their 29 COVID-19 patients with STEMI. Emergent coronary angiography confirmed culprit lesions in $60 \%$ of patients. The author emphasized that delay in reperfusion to provide adequate protection to the staff and fibrinolytic therapy may be an alternative approach. The obvious limitation of this approach is that nearly $40 \%$ will also receive fibrinolytic therapy in the absence of any underlying culprit lesion. These patients either have myocarditis or stress cardiomyopathy. In the absence of endomyocardial biopsy, it remains difficult to be sure of underlying pathology in such cases.

In the setting of non-STEMI, conservative therapy may be appropriate in low-risk patients with COVID-19 infection. High-risk patients or those with hemodynamic instability should be managed with invasive approach. ${ }^{8}$

ACS in infected patients portends a poor prognosis. Myocardial ischemia or necrosis compromises underlying functional cardiac reserve, precipitating cardiac insufficiency.

\section{Arrhythmias and Acute Heart Failure}

Patients infected with COVID-19 may present with palpitations, most commonly sinus tachycardia and, rarely, atrial fibrillation, resulting from hypoxia, inflammatory stress and abnormal metabolism. Dysrhythmias with elevated troponin levels should alert the clinician toward underlying acute myocarditis or ACS. ${ }^{10}$ Rarely, ventricular tachycardia may occur secondary to QT prolongation either as result of underlying myocarditis or some drug, namely, use of hydroxychloroquine and azithromycin.

COVID-19 infection can present with acute heart failure as the primary manifestation in $24 \%$ of patients and is associated with an increased risk of mortality. ${ }^{11}$

\section{Venous Thromboembolism}

Patients infected with the virus are also at increased risk of venous thromboembolism, resulting from systemic inflammation and abnormalities of the coagulation pathways, leading to elevations of D-dimer levels. ${ }^{10,12,13}$ In one study, all 25 patients infected with COVID-19 had elevated D-dimer levels out of which 10 patients had CT pulmonary angiography-proven pulmonary embolism. ${ }^{3}$ Low-molecular weight heparin has been used in a study to reduce mortality in severe COVID-19 infection. ${ }^{14}$

\section{COVID-19 and Chronic Cardiovascular Disease}

The increased metabolic demand as a result of infection imposes a burden on patients with underlying chronic cardiovascular disease, who have reduced cardiac reserve, leading to decompensation of stable state. Widespread systemic inflammation may be linked to increased risk of plaque rupture, worsening heart failure and stent thrombosis, and rigorous use of plaque stabilizing agents, antifailure measures and antiplatelet therapy should be considered. ${ }^{15}$

Furthermore, symptoms of COVID-19 infection tend to be more severe in these patients and a large proportion of deaths occur in patients with cardiovascular disease. The mortality may be nearly 10 times higher in these patients as compared with those without such comorbid conditions. Thus, it is important to optimally control the comorbid cardiac condition and take all preventive steps to avoid COVID-19 infection in this vulnerable group. 


\section{Conclusion}

The COVID-19 pandemic with uncertain clinical characteristics has caused an unprecedented level of burden on healthcare systems across the world. SARS-CoV-2 possibly infects the host cells through ACE2 receptors, causing myocardial damage and leading to adverse prognosis in patients with underlying cardiovascular disease. Therefore, careful monitoring of infected patients for cardiovascular complications becomes paramount to provide timely treatment in order to reduce overall mortality.

\section{Conflict of Interest}

None declared.

\section{References}

1 Wang D, Hu B, Hu C, et al. Clinical characteristics of 138 hospitalized patients with 2019 novel coronavirus-infected pneumonia in Wuhan, China. JAMA 2020;323(11):1061-1069

2 Zhou P, Yang X-L, Wang X-G, et al. A pneumonia outbreak associated with a new coronavirus of probable bat origin. Nature 2020;579(7798): 270-273

3 Huang C, Wang Y, Li X, et al. Clinical features of patients infected with 2019 novel coronavirus in Wuhan, China. Lancet 2020;395(10223):497-506

4 Clerkin KJ, Fried JA, Raikhelkar J, et al. COVID-19 and cardiovascular disease. Circulation 2020;141(20):1648-1655

5 Shi S, Qin M, Shen B, et al. Association of cardiac injury with mortality in hospitalized patients with COVID-19 in Wuhan, China. JAMA Cardiol 2020. Doi:10.1001/jamacardio.2020.0950

6 Guo T, Fan Y, Chen M, et al. Cardiovascular implications of fatal outcomes of patients with coronavirus disease 2019 (COVID-19). JAMA Cardiol 2020. Doi:10.1001/ jamacardio.2020.1017
$7 \mathrm{Hu} \mathrm{H}$, Ma F, Wei X, Fang Y. Coronavirus fulminant myocarditis saved with glucocorticoid and human immunoglobulin. Eur Heart J 2020. Doi:10.1093/eurheartj/ehaa190

8 Welt FGP, Shah PB, Aronow HD, et al; American College of Cardiology's Interventional Council and the Society for Cardiovascular Angiography and Interventions. Catheterization laboratory considerations during the coronavirus (COVID-19) pandemic: from ACC's Interventional Council and SCAI. J Am Coll Cardiol 2020;75(18):2372-2375

9 Stefanini, G, Montorfano, M, Trabattoni, D, et al. ST-elevation myocardial infarction in patients with COVID-19: clinical and angiographic outcomes Circulation 2020. Doi:10.1161/ CIRCULATIONAHA. 120.047525

10 Driggin E, Madhavan MV, Bikdeli B, et al. Cardiovascular considerations for patients, health care workers, and health systems during the coronavirus disease 2019 (COVID-19) pandemic. J Am Coll Cardiol 2020;75(18):2352-2371

11 Chen T, Wu D, Chen $\mathrm{H}$, et al. Clinical characteristics of 113 deceased patients with coronavirus disease 2019: retrospective study. BMJ 2020;368:m1091

$12 \mathrm{Wu} \mathrm{Z,} \mathrm{McGoogan} \mathrm{JM.} \mathrm{Characteristics} \mathrm{of} \mathrm{and} \mathrm{important} \mathrm{les-}$ sons from the coronavirus disease 2019 (COVID-19) outbreak in China: summary of a report of 72314 cases from the Chinese Center for Disease Control and Prevention. JAMA 2020;323(13):1239-1242

13 Ruan Q, Yang K, Wang W, Jiang L, Song J. Clinical predictors of mortality due to COVID-19 based on an analysis of data of 150 patients from Wuhan, China. Intensive Care Med 2020;46(5):846-848

14 Zhou F, Yu T, Du R, et al. Clinical course and risk factors for mortality of adult inpatients with COVID-19 in Wuhan, China: a retrospective cohort study. Lancet 2020;395(10229):1054-1062

15 Libby P, Simon DI. Inflammation and thrombosis: the clot thickens. Circulation 2001;103(13):1718-1720 